\title{
Cariología beta de tres especies pertenecientes al grupo de especies Drosophila mesophragmatica
}

\author{
Ana Beatriz Mafla M. \\ Laboratorio de Genética Evolutiva, Escuela de Ciencias Biológicas, Pontificia Universidad Católica del Ecuador. \\ Apartado 17-01-2184. Quito-Ecuador. amafla@puce.edu.ec
}

Recibido: 04, 04, 2012; aceptado: 01, 10, 2012

\begin{abstract}
RESUMEN. - Se presentan los cariotipos metafásicos de tres especies pertenecientes al grupo de especies Drosophila mesophragmatica, dos especies ecuatorianas nuevas para la ciencia: Drosophila chorlavi Céspedes \& Rafael, 2012; Drosophila rucux Céspedes \& Rafael, 2012 y la tercera D. mesophragmatica Duda, 1927 que corresponde a una población de los Andes Ecuatorianos. El número $2 \mathrm{n}=10$ es compartido por las tres especies; en los cariotipos son claramente identificables los autosomas 4 y 5 así como el cromosoma sexual $Y$; en tanto que los autosomas 2 y 3 al igual que el cromosoma sexual $X$ son prácticamente indistinguibles entre ellos. Las tres especies difieren ligeramente en el contenido de heterocromatina y marcadamente en la morfología del autosoma 5.
\end{abstract}

PALABRAS CLAVE: Cromosomas mitóticos, dípteros andinos, heterocromatina, nuevas especies.

\begin{abstract}
We present the metaphase karyotypes of three species belonging to the species group Drosophila mesophragmatica, two Ecuadorian species new to science: Drosophila chorlavi Céspedes \& Rafael, 2012, Drosophila rucux Rafael \& Céspedes, 2012 and the third D. mesophragmatica Duda, 1927 corresponds to a population of the Ecuadorian Andes. The number $2 n=10$ is shared by the three species; in the karyotypes are clearly identifiable autosomes 4 and 5 and the $Y$ sex chromosome, while the autosomes 2 and 3 as well as the sex chromosome $X$ are virtually indistinguishable from them. The three species differ slightly in heterochromatin content and markedly in autosomal morphology 5 .
\end{abstract}

KEYWORDS: Andean Diptera, heterochromatin, mitotic chromosomes, new species.

\section{INTRODUCCIÓN}

La cariología beta según White, 1978, corresponde a un segundo nivel de análisis cromosómico por el cual se consigue determinar no solo el número de cromosomas de una especie sino definir su tamaño, la posición de los centrómeros e identificar a los cromosomas sexuales; en tanto que la cariología zeta corresponde a una descripción más detallada de las bandas y otras marcas en los cromosomas politénicos (puffs, ectopic pairing, weak spots). Estos dos niveles de análisis citológico proporcionan datos complementarios para dilucidar el grado de parentesco entre dos o más especies relacionadas; más aún, en los casos en que los cromosomas politénicos resultan homosecuenciales en dos especies, la simple comparación de los bloques heterocromáticos en los cromosomas mitóticos, permite asumir qué especie es derivada de cuál otra, considerándose que la tendencia a ganar material es el carácter apomórfico (Ward y Heed, 1970). 
El grupo de especies de D. mesophragmatica fue establecido por Brncic \& Koref-Santibañez, 1957 para reunir a las especies andinas: D. mesophragmatica Duda, 1927; D. gaucha Jaeger y Salzano, 1953; D. pavani Brncic, 1957; D. altiplanica Brncic \& Koref- Santibañez, 1957; D. orkui Brncic \& Koref-Santibañez, 1957 y D. viracochi Brncic \& Koref-Santibañez, 1957.

Posteriormente, Brncic et al., 1971, proponen las relaciones citotaxonómicas entre seis especies del grupo D. mesophragmatica, $\mathrm{y}$ establecen tres unidades filéticas al interior del grupo; en su trabajo consideran de importancia dos tipos de cambios cromosómicos: las alteraciones en la cantidad de heterocromatina observada en las placas metafásicas y las inversiones paracéntricas detectadas en los cromosomas politénicos. Los autores asumen que ambos tipos de cambio ocurren al azar y se constituyen como eventos únicos en la historia evolutiva de los diferentes grupos dentro del género Drosophila, por lo que las especies que compartan estructuras cromosómicas únicas pueden ser agrupadas en unidades filéticas discretas; consecuentemente proponen que dentro del grupo mesophragmatica hay tres de estas unidades: una conformada por D. mesophragmatica, D. brncici Hunter \& Hunter, 1964 y D. gasici Brncic, 1957; otra por $D$. pavani y $D$. gaucha y una tercera sólo con $D$. viracochi. Plantean que la filogenia cromosómica propuesta requiere partir desde una secuencia de bandas en los cromosomas politénicos semejante a la de $D$. mesophragmatica y portadora del cromosoma puntiforme en el cariotipo metafásico, tal estado sería el de una Hipotética primitiva para el grupo; sugieren que esta condición "podría encontrarse en alguna población local o especie aún no descubierta...en alguna región del sistema montañoso de los Andes, propensa a mantener poblaciones aisladas y/o endémicas".

Vela y Rafael, 2004, describen tres nuevas especies del grupo D. mesophragmatica, recolectadas en el Bosque Protector del $\mathrm{Pa}$ - sochoa: D. ruminahui Vela \& Rafael, 2004; D. amaguana Vela \& Rafael, 2004; D. shyri Vela \& Rafael, 2004; en su trabajo, las autoras argumentan que la dirección de las cerdas escutelares basales es un carácter taxonómico importante, por lo cual reconocen los dos subgrupos ya propuestos por Nacrur, 1958 y proponen renombrarlos como subgrupo $D$. viracochi a las especies con cerdas escutelares anteriores convergentes y subgrupo $D$. mesophragmatica a las especies con cerdas escutelares anteriores divergentes.

Mota et al., 2008, presentan una filogenia molecular del grupo D. mesophragmatica como un ejemplo de evolución andina; dan la referencia de que otros autores ya han hecho inferencias de las relaciones dentro del grupo, basados en análisis morfológicos, citológicos, y de isoenzimas, que a nivel molecular se ha confirmado la monofilia del grupo pero que las relaciones internas no son claras, por ello argumentan que el uso de marcadores nucleares y mitocondriales podría arrojar datos esclarecedores; comparan seis de las trece especies del grupo mesophragmatica; y reportan sus resultados apoyando la subagrupación hecha por Brncic et al., 1971; reconocen tres subgrupos y entonces proponen renominarlos como subgrupos D. mesophragmatica, D. gaucha y D. viracochi; aclaran que tal subagrupación no está en discordancia con la de Vela y Rafael, 2004, pero que prefieren la de tres subgrupos pues esa topología refleja mejor sus resultados de ramificación.

Recientemente, el grupo de especies $D$. mesophragmatica se ha ampliado a 17 miembros con el descubrimiento de otras especies ecuatorianas: D. cashapamba Céspedes \& Rafael, 2012; D. chorlavi Céspedes \& Rafael, 2012; D. rucux Céspedes \& Rafael, 2012; y D. yanayuyu Céspedes \& Rafael, 2012. En esta publicación se posiciona a $D$. chorlavi como un miembro del grupo mesophragmatica mientras que a $D$. rucux se la categoriza como un "miembro aberrante" del grupo mesophragmatica. De forma que, las especies 
nuevas de este grupo de moscas andinas se constituyen en un objeto interesante para analizarlas citológicamente y complementar las propuestas mencionadas anteriormente respecto a las relaciones entre especies y entre subgrupos.

En este trabajo se reporta los resultados del análisis de los cromosomas metafásicos de Drosophila chorlavi Céspedes \& Rafael, 2012; D. rucux Céspedes \& Rafael, 2012, especies nuevas descubiertas en el Ecuador y que han sido propuestas como miembros del grupo mesophragmatica por Céspedes y Rafael, 2012; y se las compara con los cromosomas metafásicos de $D$. mesophragmatica Duda, 1927, especie de amplia distribución, desde el sur de Perú y Bolivia hasta Colombia (Koref-Santibañez, 1963).

Considerando que la comparación de bloques heterocromáticos en cromosomas mitóticos es un carácter del endofenotipo con valor citotaxonómico, creemos que la información que proporcione la cariología

\section{MATERIALES Y MÉTODOS}

Las muestras examinadas corresponden a 55 larvas de Drosophila mesophragmatica, 47 de D. rucux y 31 de $D$. chorlavi, provenientes de las colecciones que se muestran en la tabla 1 .

Para obtener cromosomas bien extendidos y diferenciados en porciones eucromáticas y heterocromáticas se utilizó la técnica de suspensión celular y la tinción con Giemsa descrita en Mafla, 2008.

Se disectaron larvas de tercer estadio para extraer los ganglios cerebrales; se colectaron de 10 a 18 ganglios cerebrales en un portaobjeto excavado con solución de Ringer-Drosophila; se transfirieron a otro portaobjeto excavado conteniendo una solución de Ringer: colchicina $0,01 \mathrm{M}$ en proporción $2: 1$ por 30 minutos; se retiró esta solución y se reemplazó por $\mathrm{KCl} 0.075 \mathrm{M}$ durante 20 minutos. Después del tratamiento hipotónico se fijó el tejido en metanol: ácido acético (3:1) durante un mínimo de 30 minutos, después de lo cual se retiró el fijador y se dispersó el tejido en ácido

Tabla 1. Datos de colección de las especies analizadas

\begin{tabular}{|c|c|c|c|c|}
\hline Especie & Colector & Localidad & $\begin{array}{l}\text { Determinado } \\
\text { por }\end{array}$ & $\begin{array}{l}\text { Código del } \\
\text { holotipo }\end{array}$ \\
\hline D. mesophragmatica & R. León & $\begin{array}{l}\text { Chimborazo: } \\
\text { Riobamba, } \\
01^{\circ} 41^{\prime} 00^{\prime \prime} S \\
78^{\circ} 38^{\prime} 00^{\prime \prime} \mathrm{W}, 2754 \mathrm{~m}\end{array}$ & V. Rafael & \\
\hline D. chorlavi & $\begin{array}{l}\text { M. L. } \\
\text { Figuero }\end{array}$ & $\begin{array}{l}\text { Ibarra: Chorlaví, } \\
0^{\circ} 21^{\prime} 30^{\prime \prime} \text {, } \\
78^{\circ} 10^{\prime} 42^{\prime \prime} \mathrm{W}, 2200 \mathrm{~m}\end{array}$ & V. Rafael & $\begin{array}{l}\text { QCAZI } \\
2316\end{array}$ \\
\hline D. rucux & $\begin{array}{l}\text { D. } \\
\text { Céspedes }\end{array}$ & 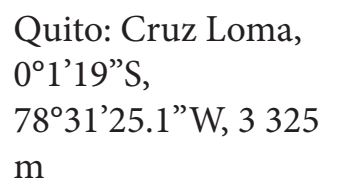 & D. Céspedes & $\begin{array}{l}\text { QCAZI } \\
2305\end{array}$ \\
\hline
\end{tabular}

beta de las tres especies ecuatorianas mencionadas, aportarían pistas para esclarecer las relaciones tanto entre especies como entre subgrupos. acético $60 \%$, se añadió dos gotas de fijador y la suspensión se goteó en portaobjetos que se conservados en etanol y en refrigeración; las placas con la suspensión se dejaron secar en una plancheta a $65^{\circ} \mathrm{C}$ (el shock térmico 
permite una mejor dispersión de los cromosomas). Luego fueron teñidas con Giemsa diluído en agua desionizada en proporción 1:20 durante 30 minutos.

Para el registro fotográfico de las placas se usó un microscopio Zeiss con cámara digital Olympus DP72; se seleccionaron los mejores núcleos para fotografiarlos con el objetivo de inmersión APO 40X y el factor 2 del Optovar obteniéndose una amplificación total de 80X. La medición de los cromosomas se hizo con el Software DP2BSW sobre las imágenes capturadas y archivadas.

En lugar de realizar cariotipos convencionales se optó por medir los cromosomas y los datos se organizaron en orden descendente. Para la identificación de la forma cromosómica se sigue las recomendaciones de Levan et al., 1964; nominando a los cromosomas m, sm, st o T de acuerdo a la posición del centrómero en la región medial, submedial, subterminal o en el punto terminal, respectivamente. Esta nomenclatura tiene la siguiente equivalencia con la nomenclatura clásica de cromosomas de Drosophila: la forma metacéntrica equivale a "V"; los submetacéntricos y subtelocéntricos a "J o barras" y los telocéntricos a "puntiformes".

Los idiogramas se construyeron normalizando los valores de 20 cariotipos (14ðð, 6 우) de D. chorlavi;

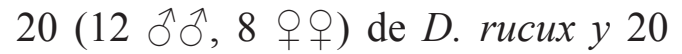
(15 ふぇ, 5 우) de D. mesophragmati$c a$. En la descripción se utiliza la Longitud Relativa (LR) definida como el valor porcentual que cada cromosoma tiene en la dotación haploide; por tanto corresponde a la relación entre la longitud de cada cromosoma frente a la suma de longitudes del complemento total.

Figura 1. Placas metafásicas de neuroblastos de Drosophila mesophragmatica a-b; D. chorlavi c-d; D. rucux e-f. La flecha sólida indica el cromosoma Y, la flecha clara al microcromosoma.

\section{RESULTADOS}

D. mesophragmatica tiene cinco pares cromosómicos: $2 \mathrm{n}=10$ (Figura 1a, b); las especies nuevas: $D$. chorlavi y D. rucux, coinciden con el cariotipo básico del grupo $D$. mesophragmatica ya que en los contajes cromosómicos de las placas mitóticas se obtuvo un número modal de 10, (87/93 en $D$. chorlavi, 40/49 en D. rucux) por lo tanto se establece que estas dos especies ecuatorianas tienen $2 n$ $=10$ (Figura. 1. c, d, e, f).

El tamaño de los cromosomas en $D$. mesophragmatica fluctúa entre 3.92 y $0.65 \mu \mathrm{m}$; en $D$. chorlavi entre 4.74 y $0.50 \mu \mathrm{m}$ y en $D$. rucux entre 3.43 y $0.81 \mu \mathrm{m}$.

En este complemento de cinco pares se identifican claramente los autosomas 4, 5 y el cromosoma sexual $Y$. El autosoma 4 es el par más grande, de longitud relativa (LR) 15.60 en D. mesophragmatica, 16.98 en D. chorlavi

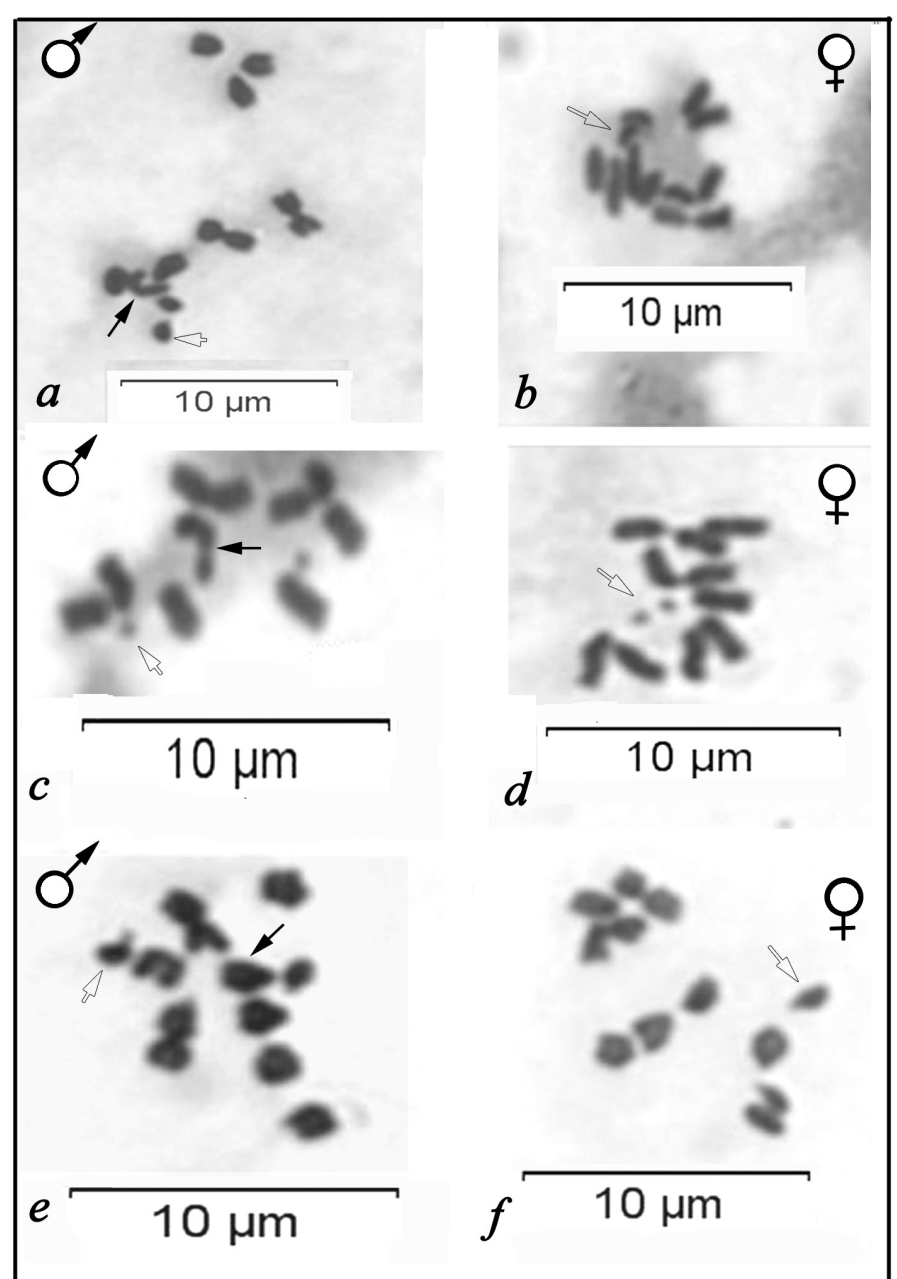


y 15.81 en D. rucux; tiene el centrómero en la región medial por lo que se le puede identificar como metacéntrico en las tres especies. El más pequeño del complemento es el autosoma 5, claramente heterocromático; en $D$. chorlavi es puntiforme o telocéntrico de LR 2.85; en tanto que en $D$. mesophragmatica y D rucux es submetacéntrico de LR 6.36 y 6.66, respectivamente. El cromosoma $Y$ también se distingue con claridad por su magnitud y heteropicnosis, en orden descendente ocupa el segundo lugar en longitud; en $D$. chorlaví es subtelocéntrico y con un satélite conspicuo en el brazo largo, LR 13.15; al contrario que el de D. rucux, cuyo aspecto es semejante al de D. mesophragmatica, submetacéntrico y sin satélite visible, LR
11.16 en la primera especie y 12.21 en la segunda especie Figura $2 \mathrm{~d}$.

El cromosoma $X$ así como los pares autosómicos 2 y 3 son indistinguibles entre ellos, tanto en tamaño como en morfología; los tres aparecen como submetacéntricos, los brazos cortos son totalmente heterocromáticos y de LR: 10.31, 8.96, 8.06 en D. mesophragmatica; $11.07,9.77,8.84$ en D. chorlavi y 10.12, 9.09, 8.24 en D. rucux. En placas excepcionales se pudo reconocer el par heteromórfico, y definir que el cromosoma $X$ ocupa el tercer lugar en orden descendente de longitud (Figura $2 a, b$, c, d). Por lo tanto el par $X Y$ es heteromórfico: sm-st el $Y$ de mayor longitud.
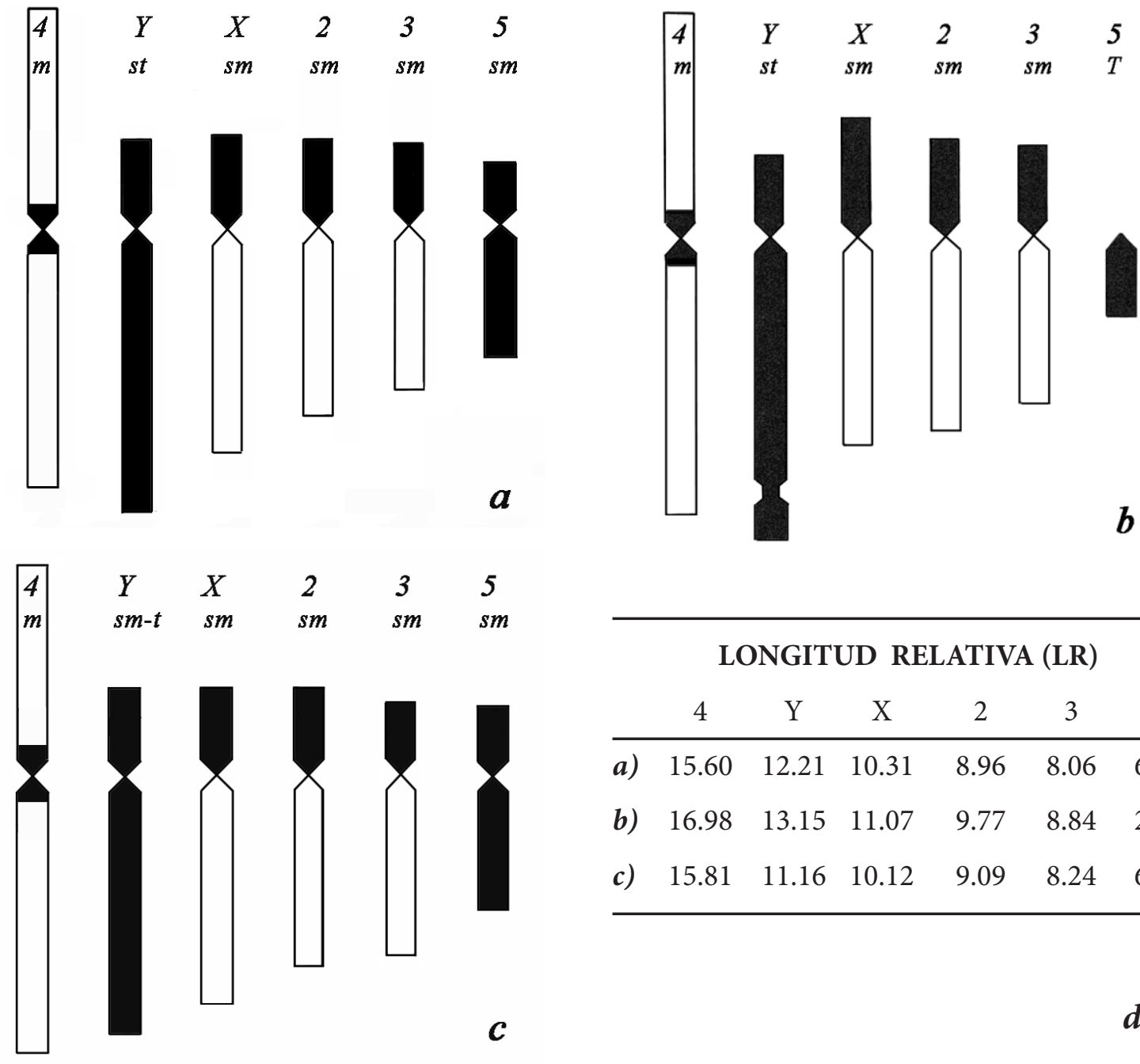

LONGITUD RELATIVA (LR)

\begin{tabular}{ccccccc} 
& 4 & $\mathrm{Y}$ & $\mathrm{X}$ & 2 & 3 & 5 \\
\hline a) & 15.60 & 12.21 & 10.31 & 8.96 & 8.06 & 6.36 \\
b) & 16.98 & 13.15 & 11.07 & 9.77 & 8.84 & 2.85 \\
c) & 15.81 & 11.16 & 10.12 & 9.09 & 8.24 & 6.66 \\
\hline
\end{tabular}

$d$

Figura 2. Idiogramas de las tres especies: a) Drosophila mesophragmatica; b) D. chorlavi; c) d. rucux y d) Valores de Longitud Relativa (LR) usados en los idiogramas. 
La distribución de heterocromatina es semejante en las tres especies: el autosoma 4 más grande tiene un contenido de $2 \%$ de heterocromatina en la región pericentromérica, los brazos cortos del cromosoma sexual $X$, así como los de los autosomas 2 y 3, fluctúan entre 2.41 y $3.96 \%$, en tanto que la totalidad del cromosoma sexual $Y$ así como del pequeño autosoma 5 son completamente heterocromáticos. En los idiogramas de las tres especies (Figura 2) se aprecia claramente las porciones eucromáticas y heterocromáticas; en total, cada una de las tres especies tienen alrededor de un tercio del genoma haploide estructurado en heterocromatina (Tabla 2, Figura 2).
En suma, los cariotipos mitóticos de $D$. rucux y $D$. mesophragmatica son muy parecidos entre sí y diferentes al de D. chorlavi (Figura $2 \mathrm{a}, \mathrm{b}, \mathrm{c})$.

\section{DISCUSIÓN}

La apariencia de las placas metafásicas en las tres especies es semejante a lo reportado por Brncic y Koref-Santibañez, 1957 como característico del grupo D. mesophragmatica, es decir comprende 1 par en V, 3 pares de barras y 1 par puntiforme. La forma de pequeña barra del par 5, igualmente ha sido reportada en algunos miembros del grupo como $D$. mesophragmatica, D. altiplánica y D. orkui por

Tabla 2. Distribución de la heterocromatina en valores porcentuales

\begin{tabular}{clccc}
\hline Cromosoma & Heterocromatina & $\begin{array}{c}\text { Drosophila } \\
\text { mesophragmatica }\end{array}$ & chorlaví & rucux \\
\hline 4 & pericentromérica & 2.00 & 2.00 & 2.00 \\
$\mathrm{X}$ & brazo corto & 2.98 & 3.96 & 2.78 \\
2 & brazo corto & 2.79 & 3.31 & 2.83 \\
3 & brazo corto & 2.62 & 3.09 & 2.41 \\
5 & todo & 6.36 & 2.83 & 6.66 \\
$\mathrm{Y}$ & todo & 12.21 & 13.15 & 11.16 \\
& Total: & 28.96 & 28.24 & 27.84 \\
\hline
\end{tabular}

Las diferencias más notables entre las nuevas especies ecuatorianas: D. chorlavi y D. rucux son dos: 1) el tamaño y morfología del autosoma 5 , en la primera es telocéntrico y pequeño de LR $2.85 \%$ mientras que en la segunda es submetacéntrico y más grande de LR $6.66 \%$. 2) La morfología del cromosoma $Y$, en la primera es subtelocéntrico y con una constricción secundaria bien definida en el brazo largo; en tanto que en D. rucux es submetacéntrico (el índice centromérico de 25.5 corresponde a la cifra límite usada para definir la morfología como submetacéntrico o subtelocéntrico) y no se visualiza ninguna constricción secundaria.
Brncic et al., 1971, de modo que al compartir el carácter $2 \mathrm{n}=10$ las especies ecuatorianas D. chorlavi y D. rucux están relacionadas con los otros miembros del grupo, por lo tanto acertadamente incluidas en el grupo D. mesophragmatica (Céspedes y Rafael, 2012).

Respecto a la morfología y tamaño del cromosoma 5 diferente en $D$. chorlavi y D. rucux, se ve la misma relación registrada por Brncic et al., 1971 entre las especies del subgrupo $D$. mesophragmatica y las del subgrupo D. pava$n i$; diferencia que les llevó a proponer la reunión de las especies que tienen el cromosoma 5 "alargado" y que son: D. mesophragmatica, D. gasici, D. brncici en una unidad filética; separadas de otra unidad portadora del 5 puntiforme 
y que corresponde a las especies: D. gaucha, $D$. pavani y $D$. viracochi. En esta línea deductiva: D. chorlavi podría ubicarse como un miembro más tanto del subgrupo gaucha como del subgrupo viracochi (según Mota, 2008) y D. rucux dentro del subgrupo mesophragmatica.

Como se ha señalado antes, la cariología beta ha de ser complementada con la cariología zeta para obtener evidencias que establezcan los nexos de parentesco, en el caso de las nuevas especies falta este complemento aun cuando se cuenta con datos preliminares: el cariotipo de $D$. chorlavi fue reportado (Romero, 2010) como estructuralmente similar a los de las especies del grupo D. mesophragmatica, es decir que posee 5 filamentos largos: X, II, III, IV-R, IV-L, con telómeros bien definidos y extremos basales que coalescen en un cromocentro heteropicnótico que incluye al pequeño $\mathrm{V}$; así mismo registró lazos de inversión en los cromosomas X, II, IV-R, IV-L, y además un segmento duplicado, que comprende las secciones 60 a la 75 del cromosoma IV R; sin embargo tales reestructuras cromosómicas no se han observado en las preparaciones de Salas, 2011 quien construyó el mapa de los cromosomas politénicos de $D$. chorlavi en la que se muestra una secuencia de bandas similar a la de D. pavani; con lo dicho, se podría asumir que esta especie puede ser ubicada en el subgrupo D. gaucha. No obstante a Céspedes y Rafael, 2012 les llama la atención el aspecto "atigrado" de D. chorlavi "que la acerca a las especies del grupo repleta", así como la convergencia de las cerdas escutelares, carácter considerado plesiomórfico; en consecuencia parecería apropiada la integración que Céspedes y Rafael hacen de $D$. chorlavi dentro del subgrupo viracochi. Es claro que la subagrupación ha de continuar siendo preliminar hasta contar con la información que proporcionen los estudios comparativos de cromosomas politénicos de $D$. viracochi y de $D$. shyri.

Con respecto a la posición de $D$. rucux, con el sustento de que la convergencia citológica es extremadamente improbable en dos linajes, parece apropiado considerarla en el subgrupo mesophragmatica, junto a las especies que poseen el cromosoma 5 alargado.

La subagrupación realizada por Brncic et al., 1971 así como la de Mota et al., 2008 coinciden y refuerzan la idea de usar la comparación de cantidad de heterocromatina en la distinción de linajes. La importancia de la cantidad de heterocromatina parece ser su relación con el ADN satélite, así como con los elementos transponibles y el papel que juega en la especiación; así lo establece la hipótesis de Korochkiri, 1983 quien supone que las diferencias en DNA satélite dependen de la afinidad del genoma con los "genes saltarines" y que estos genes pueden determinar la redistribución de heterocromatina provocando cambios en los procesos moleculares y morfogenéticos durante el desarrollo, por lo tanto: la afinidad del genoma por genes saltarines específicos puede cambiar por una simple mutación la cual correspondería a una "gran mutación", que podría determinar el origen de una nueva especie por un salto y no por la acumulación de pequeños cambios, como lo propuso Goldschmidt (citado por Gould, 1980). Esta hipótesis se complementa con el trabajo de Pimpinelli et al., 1993 y muchos otros en la misma línea, que muestran a los elementos transponibles como componentes estructurales de la heterocromatina.

\section{CONCLUSIONES}

- Las especies ecuatorianas D. chorlavi y D. rucux comparten el número cromosómico $2 \mathrm{n}=10$ con las especies que conforman el grupo de especies $D$. mesophragmatica.

- D. chorlavi y D. rucux difieren entre ellas en el tamaño y morfología del cromosoma 5; esta característica se considera como indicio para posicionar a $D$. chorlavi en uno de los subgrupos que tienen el autosoma 5 puntiforme (D. gaucha $o$ D. viracochi) y a D. rucux en el subgrupo D. mesophragmatica. 


\section{AGRADECIMIENTOS}

A la Pontificia Universidad Católica del Ecuador por financiar los proyectos: "Cariología $\mathrm{Z}$ de nuevas especies del subgrupo inca" $\mathrm{E}$ 29179 y "Cariología Z de nuevas especies de Drosophila" G19115. A la Dra. Violeta Rafael por la provisión de las muestras de estas especies así como por sus entusiastas comentarios y por fomentar la interdisciplinariedad. Al Dr. Clifford Keil por sus valiosas acotaciones al manuscrito. A la Dra. Laura Arcos ex Decana de la Facultad de Ciencias Exactas y Naturales por su permanente apoyo a la investigación.

\section{REFERENCIAS BIBLIOGRÁFICAS}

Brncic D y Koref-Santibañez S. 1957. The Mesophragmatica Group of Species of Drosophila. Evolution, 11:300-310.

Brncic D, Nair PS y Wheeler MR. 1971. Cytotaxonomic Relationships Within the mesophragmatica Species Group of Drosophila. STUDIES IN GENETICS VI. University of Texas Publications, 7103.

Céspedes D y Rafael V. 2012. Cuatro especies nuevas del grupo de especies Drosophila mesophragmatica (Diptera, Drosophilidae) en los Andes ecuatorianos. Iheringia, Série. Zoologia, 102(1).

Gould SJ. 1983. El regreso del monstruo esperanzado. En GOULD, S. J. El Pulgar del Panda: 197-205. Hermann Blume Ediciones. Primera edición española Fuenlabrada, Madrid.

Koref-Santibañez S. 1963. Courtship and Sexual Isolation in Five Species of the $\mathrm{Me}$ sophragmatica Group of the Genus Drosophila. Evolution, 17(1): 99-106.

Korochkiri LI. 1983. Institute of Developmental Biology, Moscow, USSR. The hypothesis about the role of heterochromatin in the evolution of Drosophila of the virilis group.DIS, 59:70.
Levan A, Fredga K y Sandberg AA. 1964. Nomenclature for centromeric position on chromosomes. Hereditas, 52(2).

Mafla AB. 2008. Cariotipos metafásicos de Drosophila inca y D. yangana, subgrupo inca, grupo repleta. Revista Ecuatoriana de Medicina y Ciencias Biológicas, XIX(1 y 2).

Mota NR, Robe LJ, Vera LS, Budnik VM y Loreto E. 2008. Phylogeny of the Drosophila mesophragmatica group (Diptera, Drosophilidae): An Example of Andean Evolution. Zoological Science, 25: 526532.

Pimpinelli S, Berloco M, Fanti L, Dimitri P, Bonaccorsi S, Marchetti E, Caizzi R, Caggese C y Gatti M. 1993. Transposable elements are stable structural components of Drosophila melanogaster heterochromatin. Proceedings of the National Academy of Sciences, 92:3804-3808.

Romero G. 2010. Informe de avance del Proyecto "Cariología $\mathrm{Z}$ de nuevas especies del subgrupo inca" E 29179.

Salas ISA. 2011. Caracterización de los cromosomas politénicos de una nueva especie de Drosophila perteneciente al grupo mesophragmatica. Tesis de Licenciatura, Pontificia Universidad Católica del Ecuador. Quito, Ecuador.

Vela D y Rafael V. 2004. Three new Andean species of Drosophila (Diptera, Drosophilidae) of the mesophragmatica group. Iheringia Série Zoologia, 94(3):295-299.

White MJD. 1978. Modes of Speciation. W.H. Freeman and Company, San Francisco.

Ward BL y Heed WB. 1970. Chromosome Phylogeny of Drosophila pachea and Related Species. Journal of Heredity, 61(6): 248-258. 